\title{
Estágios motivacionais, sintomas de ansiedade e de depressão no tratamento do tabagismo
}

\author{
Wilson Vieira Melo \\ FACCAT - Faculdades de Taquara \\ Margareth da Silva Oliveira \\ Pontifícia Universidade Católica do Rio Grande do Sul \\ Elisa Arrenti Ferreira \\ WP - Centro de Psicoterapia Cognitivo-Comportamental
}

\begin{abstract}
RESUMO
O tabagismo é um problema de saúde pública que tem feito diversas vítimas ao longo dos anos (Gigliotti, Carneiro \& Ferreira, 2001). Uma das principais dificuldades encontradas pelos fumantes quando tentam parar de fumar é a motivação (Heather, 1992; Di Clemente \& Prochaska, 1985). O objetivo desse estudo quase-experimental foi avaliar a mudança nos estágios motivacionais ao longo das oito semanas de tratamento do tabagismo. A amostra foi composta por 109 sujeitos avaliados em 4 momentos. Os instrumentos utilizados foram as Escalas URICA, BAI, BDI, FTND e o Monoxímetro. Os resultados indicam que os escores de ansiedade e de depressão daqueles que aderiram ao tratamento foram menores do que quando comparados àqueles que não aderiram $(p=0,023) \mathrm{e}$ $(p=0,022)$, respectivamente. Ademais, apenas a sub-escala da "Manutenção", apresentou aumento significativo $(\mathrm{p}<0,001)$, uma vez que as demais mantiveram as mesmas médias de pontos. Esse aumento não está relacionado com o nível de dependência de nicotina $(p=0,244)$. O nível de dependência de nicotina também não demonstrou estar relacionado à aderência ao tratamento $(p=0,687)$. Discute-se a importância de se avaliar os estágios motivacionais, a ansiedade e a depressão em tabagistas, pois esses parecem ser fundamentais nesse contexto.
\end{abstract}

Palavras-chave: tabagismo; estágios motivacionais; avaliação.

\begin{abstract}
Motivational stages, anxiety and depression symptoms during smoking treatment

Smoking is a public health problem that has victimized many people along the years (Gigliotti, Carneiro \& Ferreira, 2001). Motivation is one of the most difficult aspects smokers have to face when they try to stop smoking (Heather, 1992; Di Clemente \& Prochaska, 1985). The objective of this quasi-experimental study was to evaluate change in motivational stages during an eight-week smoking cessation treatment. The sample was comprised of 109 subjects who were evaluated in 4 moments, using the URICA, BAI, BDI, FTND Scales and the Monoximeter. The results indicate that the anxiety and depression scores of those who adhered to the treatment were lower than the ones of those who did not ( $p=0,023$ and $p=0,022$, respectively). In addition, only the sub-scale of "maintenance" showed a significant increase in score $(p<0,001)$, since the other ones maintained the same mean scores. Such increase is not related to the nicotine dependence level $(p=0,244)$. This level did not have any correlation to joining the treatment $(p=0,687)$. The importance in evaluating motivational stages, anxiety and depression in smokers are discussed, for they seem to be fundamental in this context.
\end{abstract}

Keywords: smoking; motivational stages; evaluation.

O Modelo Transteórico, que foi proposto pelos psicólogos James O. Prochaska e Carlo C. Di Clemente (1982), vem sendo utilizado em larga escala nos últimos 20 anos em estudos relacionados aos comportamentos aditivos tais como uso de drogas, álcool, comportamento de risco para HIV/AIDS e dietas, além do tabaco (Burke, Arkowitz \& Menchola, 2003). Este último é um dos maiores responsáveis pela causa e agravamento de doenças em milhões de pessoas que consomem esta toxina diariamente em 
todo o mundo (Gigliotti, Carneiro \& Ferreira, 2001). Seu uso também traz a morte prematura a um grande número de pessoas a cada ano, além de contribuir para a invalidez e para o aumento do sofrimento em outras tantas. Aproximadamente um terço de todos os usuários de tabaco no mundo morrerão prematuramente por causa de sua dependência. Ao contrário de muitas epidemias no passado, há uma compreensão da causa desta morte prematura - o uso do tabaco (Achutti, 2001; Gigliotti e cols., 2001; Gigliotti e cols., 1999).

Embora existam evidências acerca da validade e da importância do Modelo Transteórico, alguns aspectos do construto não foram bem validados e, por isso, mais análises teóricas são necessárias (Pollak, Carbonari, Di Clemente, Niemann \& Mullen, 1998; Sutton, 1997). Esse modelo propõe uma descrição de como as pessoas modificam seus comportamentos aditivos, com ou sem tratamento, tratando a motivação, fundamental nesse contexto (Heather, 1992), como um estado de prontidão ou de avidez para a mudança (Prochaska \& Di Clemente, 1982). Para os autores, as pessoas atravessam cinco estágios motivacionais, que são: 1) Pré-Contemplação; 2) Contemplação; 3) Preparação; 4) Ação e 5) Manutenção (Di Clemente \& Prochaska, 1985).

Na "Pré-Contemplação", as pessoas não acreditam que devam mudar seu comportamento e nem estão dispostas a realizar algo a respeito, em face de que não têm nenhum desejo o fazê-lo. Já no estágio da "Contemplação", as pessoas, apesar de darem-se conta de que deveriam mudar seu comportamento, ainda não estão sentindo-se preparadas para realizar essa mudança e não estão tentando nada efetivamente. O estágio da Preparação, um dos aspectos do construto teórico que mais vem recebendo críticas nos últimos anos, diz respeitos àquelas pessoas que estão planejando os passos para sua futura mudança. A crítica a esse estágio se dá devido à dificuldade de se investigar empiricamente esse desejo de mudança. De um modo geral, os instrumentos utilizados para avaliação dos estágios motivacionais não levam em consideração esse ponto (Carey, Purnine, Maisto \& Carey, 1999). O estágio da "Ação" diz respeito às pessoas que já começaram a fazer alguma coisa para modificar seu comportamento problema. Finalmente, o estágio da "Manutenção" é aquele em que as pessoas têm sustentadas as suas mudanças comportamentais mas ainda estão cientes de que existe um risco de recaída (Di Clemente \& Prochaska, 1985).

Diversos estudos têm sido realizados com pacientes tabagistas na tentativa de se mapear possíveis variá- veis envolvidas no processo de parada do uso de tabaco. Nesse contexto, podem-se citar os sintomas de ansiedade e de depressão como sendo um dos fatores mais significativos (Fergusson, Goodwin \& Horwood, 2003; O'Connell e cols., 2003). A ansiedade é um dos sintomas mais comumente encontrados na síndrome de abstinência da nicotina e o seu aumento implica um dos principais fatores de risco de recaída (O'Connell e cols., 2003). Por outro lado, alguns importantes estudos têm demonstrado que quanto maior a presença de sintomas de depressão, maior a dificuldade em se conseguir atingir a abstinência efetiva, no que se refere ao tratamento do tabagismo (Gigliotti e cols., 2001; Fergusson e cols., 2003).

Este artigo tem o objetivo de apresentar um estudo de avaliação dos estágios de motivação para mudança, sintomas de ansiedade e de depressão, associando-os com a manutenção da abstinência de tabaco após intervenção breve para cessação do consumo dessa substância.

\section{MÉTODO}

Trata-se de um estudo clínico onde foi feita uma avaliação pré e pós-intervenção, do grupo em comparação com ele mesmo. Como não se encontrou informações acerca de estudos anteriores, calculou-se uma amostra com $95 \%$ de confiança e $10 \%$ de erro, maximizando a variância. Desta forma, chegou-se ao número de 96 indivíduos. No decorrer do trabalho, ingressaram todos os pacientes que preencheram os critérios de inclusão. Ao termino do período, obtevese a informação de 109 indivíduos, tendo atingido o tamanho da amostra exigido.

\section{Participantes}

A amostra foi de conveniência, composta por 109 pacientes fumantes residentes em Porto Alegre e na região metropolitana, que buscaram tratamento espontaneamente no Ambulatório de Auxílio ao Abandono do Tabagismo do Hospital São Lucas da PUCRS durante o período de outubro de 2003 a outubro de 2004.

Foram critérios de exclusão, ter menos de 18 anos, ter escolaridade inferior à $5^{\mathrm{a}}$ série do Ensino Fundamental e ser dependente ou abusador de outras substâncias psicoativas, exceto a cafeína. Os critérios de inclusão foram de que o paciente esteja entrando em contato pela primeira vez com o serviço e estar fumando atualmente. 


\section{Instrumentos}

Todos os pacientes foram avaliados através de entrevista estruturada para obtenção de dados sociodemográficos e para a confirmação diagnóstica de dependência de tabaco com consumo atual.

Para verificar os estágios motivacionais, em relação ao uso de tabaco, foi utilizada a escala University of Rhode Island Change Assesment Scale - URICA (Mc Connaughy, Di Clemente, Prochaska \& Velicer, 1983), que é composta por 32 itens divididos nas sub-escalas: Pré-contemplação, Contemplação, Ação e Manutenção. Essa escala pode ser utilizada para avaliação de qualquer comportamento aditivo, já tendo sido validada e adaptada para a população brasileira (Figlie, 1999).

O Beck Depression Inventory - BDI (Beck \& Steer, 1993a) e o Beck Anxiety Inventory - BAI (Beck \& Steer, 1993b) são escalas desenvolvidas para avaliação dos sintomas de depressão e de ansiedade, tanto em pacientes psiquiátricos como na população em geral. Ambas foram validadas para a população brasileira por Cunha (2001).

A avaliação da dependência de tabaco foi feita através do uso da escala Fagerström Tolerance of Nicotine Dependence - FTND (Fagerström, 1978). Esta é um questionário de seis itens auto-aplicável e tem a finalidade de determinar o grau de dependência de nicotina. Uma pontuação alta indica maior gravidade de dependência do tabaco. Neste estudo foi aplicada a validação brasileira (Carmo \& Pueyo, 2002), e os pontos de corte utilizados foram os publicados por Achutti (2001), já que esse estudo não os apresenta.

A medida da intoxicação por Monóxido de Carbono (CO) foi verificada pelo uso do Monoxímetro. Esse é um aparelho de fácil manejo que permite a medida do CO através da expiração no bocal do aparelho, posterior a uma apnéia forçada de 15 segundos. As concentrações de $\mathrm{CO}$ são medidas após a expiração e aparecem imediatamente no visor. Esse aparelho mede o $\mathrm{CO}$ em partículas por milhão (ppm). Como uma regra geral, os níveis de $\mathrm{CO}$ no ar ambiente alcançam algo em torno de $04 \mathrm{ppm}$. Os níveis médios para fumantes são perto de $33 \mathrm{ppm}$ e nos fumantes chegam a ultrapassar esses níveis. Os valores de 0 a $10 \mathrm{ppm}$ podem ser obtidos em pessoas não fumantes, os níveis entre $11-20 \mathrm{ppm}$ de $\mathrm{CO}$, correspondem à intoxicação por $\mathrm{CO}$ de um fumante moderado e os níveis entre 21-100 ppm de $\mathrm{CO}$, a fumantes graves (Bedfont Scientific, 1993). Essas medidas vão aumentando durante o dia, enquanto se fuma, e podem permanecer altas na corrente sangüínea por 24 horas, dependendo de vários fatores, tais como atividades físicas, sexo, variação individual e intensidade de inalação.

\section{Procedimentos}

Os participantes passaram por um acompanhamento de oito semanas onde, durante esse período, foram realizadas quatro medidas da escala URICA, a fim de se realizar o monitoramento dos estágios motivacionais para averiguar se este está associado com a manutenção da abstinência de tabaco após intervenção breve para cessação do consumo dessa substância. Foi avaliado o grau de dependência de nicotina da amostra e também foi realizada uma avaliação do tipo pré e pós dos índices de ansiedade e de depressão, bem como dos níveis de intoxicação por CO.

Ao chegar para triagem no Ambulatório de Auxílio ao Abandono do Tabagismo do Hospital São Lucas da PUCRS, todos os pacientes que preencheram os critérios de inclusão e aceitaram participar do estudo, foram submetidos à aplicação dos inventários BAI, BDI e da escala URICA e assistiram a uma reunião informativa sobre o tabagismo, coordenada por uma estagiária do curso de Enfermagem. Ao cabo dessas duas primeiras etapas, os pacientes passaram por uma consulta com os médicos do Ambulatório, onde foi preenchido o questionário padrão, medido o $\mathrm{CO}$ através do Monoxímetro e aplicada a FTND. Foi prescrita a medicação aos pacientes, com orientações acerca do tratamento. As medicações utilizadas foram a bupropiona $300 \mathrm{mg}$, nortriptilina $75 \mathrm{mg}$, além de adesivos transdérmicos de 21, 14 e $7 \mathrm{mg}$ e gomas de mascar de reposição de nicotina. A escolha da farmacoterapia bem como das doses e tempo de utilização variavam de acordo com as necessidades específicas e limitações clínicas de cada paciente, e também dependiam do aparecimento de efeitos colaterais.

Na segunda e terceira consulta, foi feito o acompanhamento medicamentoso dos pacientes pela equipe do ambulatório e foi reaplicada a escala URICA para avaliar se a primeira intervenção realizada, ou seja, aplicação de escalas mais consulta médica, já havia causado algum tipo de impacto na motivação desses pacientes. Ao cabo das oito semanas, os pacientes foram submetidos à quarta medida da Escala URICA, além de sofrer nova avaliação quanto aos níveis de ansiedade, de depressão e de intoxicação por CO. Os custos financeiros com a compra da medicação para o tratamento foram custeados na sua totalidade pelos próprios pacientes. 
Essa pesquisa foi submetida à apreciação pelo Comitê de Ética em Pesquisa da Pontifícia Universidade Católica do Rio Grande do Sul e obteve aprovação em setembro de 2003. Todos os procedimentos foram detalhadamente explicados aos pacientes, incluindo os riscos que por ventura possam estar envolvidos. O Termo de Consentimento Livre e Esclarecido foi lido e as dúvidas dirimidas. Frente à aceitação do sujeito, o Termo foi devidamente assinado em duas vias, uma permanecendo com o paciente e outra com o pesquisador.

\section{Análise dos dados}

As informações coletadas nesta pesquisa foram organizadas na forma de Banco de Dados no Progra- ma "Statistical Package for the Social Sciences" (SPSS) versão 11.5.1. Foi realizada estatística descritiva para uma análise exploratória dos dados, através de média, desvio padrão, freqüência e percentual. Para realizar a análise inferencial, utilizou-se os testes estatísticos de Análise de Variância com medidas repetidas, teste de Friedman, teste exato de Fisher, teste de Mann-Whitney e o teste de Wilcoxon, sendo adotado um nível mínimo de significância de 5\%.

\section{RESULTADOS}

A amostra foi composta por 109 sujeitos, com média de idade de 48,28 $(\mathrm{SD}=11,63)$ anos. A renda familiar média foi de $\mathrm{R} \$ 1.560,17$ ( $\mathrm{SD}=1.828,02)$.

Tabela 1: Dados sociodemográficos da amostra

\begin{tabular}{|c|c|c|}
\hline Variáveis & $\mathbf{N}$ & $\%$ \\
\hline \multicolumn{3}{|l|}{ Sexo } \\
\hline Homens & 30 & $27,5 \%$ \\
\hline Mulheres & 79 & $72,5 \%$ \\
\hline \multicolumn{3}{|l|}{ Fonte de Encaminhamento } \\
\hline SUS & 82 & $75,2 \%$ \\
\hline Convênios & 16 & $14,7 \%$ \\
\hline Semi-Privativo & 11 & $10,1 \%$ \\
\hline \multicolumn{3}{|l|}{ Estado Civil } \\
\hline Solteiro(a) & 16 & $14,7 \%$ \\
\hline Casado(a) & 59 & $54,1 \%$ \\
\hline Separado(a) & 25 & $22,9 \%$ \\
\hline Viúv (a) & 09 & $8,3 \%$ \\
\hline \multicolumn{3}{|l|}{ Escolaridade } \\
\hline Ensino Fundamental Incompleto & 33 & $30,3 \%$ \\
\hline Ensino Fundamental & 15 & $13,8 \%$ \\
\hline Ensino Médio Incompleto & 06 & $5,5 \%$ \\
\hline Ensino Médio & 24 & $22 \%$ \\
\hline Curso Técnico / Profissionalizante & 07 & $6,4 \%$ \\
\hline Ensino Superior Incompleto & 07 & $6,4 \%$ \\
\hline Ensino Superior & 17 & $15,6 \%$ \\
\hline \multicolumn{3}{|l|}{ Situação Profissional / Ocupacional } \\
\hline Do lar & 16 & $14,7 \%$ \\
\hline Autônomo(a) & 16 & $14,7 \%$ \\
\hline Com vínculo empregatício & 43 & $39,4 \%$ \\
\hline Desempregado(a) & 08 & $7,3 \%$ \\
\hline Aposentado(a) & 26 & $23,9 \%$ \\
\hline \multicolumn{3}{|l|}{ Prática Religiosa } \\
\hline Sim & 57 & $52,3 \%$ \\
\hline Não & 52 & $47,7 \%$ \\
\hline Total & 109 & $100 \%$ \\
\hline
\end{tabular}


No que diz respeito ao perfil dos fumantes quanto ao tempo de fumo, 20,2\% $(\mathrm{n}=22)$ diziam fumar há mais de 40 anos e $26,6 \%(n=29)$ fumavam de 31 a 40 anos. Além disso, 39,4\% $(\mathrm{n}=43)$ disseram fumar de 21 a 30 anos, o que quer dizer que $86,2 \%(n=94)$ dessa amostra fumava há mais de 20 anos. Se usarmos o ponto de corte de 10 anos ou mais, esse índice sobe para $97,2 \%(\mathrm{n}=106)$ visto que $11 \%(\mathrm{n}=12)$ fumava de 11 a 20 anos. Apenas $2,8 \%(n=3)$ dos pacientes disseram fumar há menos de 10 anos.

Todos os tabagistas fumavam exclusivamente cigarro, isto é, não foi encontrado ninguém que fumasse somente charuto, palheiro ou cachimbo nessa amostra. No que se refere à quantidade de cigarros fumada, nessa amostra, 9,2\% (n=10) diziam fumar mais de 40 cigarros/dia. Ademais, 24,8\% ( $\mathrm{n}=27)$ fumavam de 31 a 40 cigarros/dia, $21,1 \% \quad(n=23)$ de 21 a 30 cigarros/dia, 37,6\% $(\mathrm{n}=41)$ de 11 a 20 cigarros/dia e apenas $7,3 \%(\mathrm{n}=8)$ dos pacientes fumavam menos que 10 cigarros diários. A média de tentativas prévias para se parar de fumar nessa amostra foi de 3,04 $(\mathrm{SD}=3,12)$.

Também foi investigado o uso de outras substâncias psicoativas pelos pacientes que compunham essa amostra. O uso foi dividido em "nunca usou", "uso na vida", "uso no último ano" e "uso atual". Casos de abuso ou dependência de outras substâncias foram excluídos da amostra dados os critérios de exclusão. Assim, no que se refere ao consumo de álcool, 17,4\% $(\mathrm{n}=19)$ disse nunca ter usado, $21,1 \%(\mathrm{n}=23)$ já fez uso na vida, $0,9 \%(n=1)$ fez uso no último ano e $60,6 \%$ $(\mathrm{n}=66)$ faz uso atualmente de álcool.

O uso de substâncias benzodiazepínicas teve 5,5\% $(n=6)$ da amostra fazendo uso no último ano e aqueles que estavam usando este tipo de substância atualmente, $11 \%(n=12)$, foram excluídos da amostra. Os que afirmaram nunca terem usado esse tipo de medicação somaram 66,1\% (n=72) da amostra e os que já fizeram uso na vida somaram $17,4 \%(n=19)$. No que se refere ao uso de cannabinóides, $11,9 \%(\mathrm{n}=13)$ disse ter usado na vida e $88,1 \%$ ( $n=96$ ) afirmou nunca ter experimentado.

Quanto ao uso de cocaína, crack ou merla, 3,7\% $(n=4)$ fez uso na vida e $96,3 \%(n=105)$ afirmou nunca ter experimentado. No que se refere a anfetaminas e outras drogas sintéticas $12,8 \%(\mathrm{n}=14)$ fizeram uso na vida, $1,8 \%(\mathrm{n}=2)$ no último ano e $85,3 \%(\mathrm{n}=93)$ afirmaram nunca ter experimentado. Quanto aos inalantes, $2,8 \%(\mathrm{n}=3)$ disse ter utilizado na vida, $0,9 \%(\mathrm{n}=1)$ fazia uso atualmente e $96,3 \%(n=105)$ nunca ter utilizado. Opióides foram utilizados na vida por $0,9 \%$ $(\mathrm{n}=1)$ da amostra e os alucinógenos e asteróides ana- bolizantes não foram utilizados por nenhum dos pacientes que compuseram essa amostra.

A amostra foi dividida em dois grupos distintos, isto é, aqueles que aderiram e aqueles que não aderiram ao tratamento. Os pacientes que compõem o grupo dos que foram considerados aderentes ao tratamento totalizam $23,9 \% \quad(n=26)$ da amostra e são aqueles que completaram todas as etapas da avaliação, ou seja, ficaram vinculados ao serviço do Ambulatório de Auxílio ao Abandono do Tabagismo por pelo menos oito semanas. Aqueles que não completaram as quatro medidas da Escala URICA, nem as medidas de antes e depois de ansiedade, depressão e intoxicação por $\mathrm{CO}$ foram considerados não aderentes ao tratamento e compunham $76,1 \%(n=83)$ da amostra estudada.

Para se avaliar o grau de dependência de nicotina desses pacientes, foi utilizada a Escala FTND que apontou que 33\% ( $n=34)$ da amostra tiveram índices de dependência graves, $53,4 \%(\mathrm{n}=55)$ índices moderados e $13,6 \%(n=14)$ índices leves, com uma média de 6,25 pontos $(\mathrm{SD}=2,23)$, situada na categoria de dependência moderada. Isso indica que $86,4 \%$ possuíam dependência moderada ou grave nessa amostra.

O uso do Monoxímetro possibilitou uma medida objetiva dos níveis de intoxicação por $\mathrm{CO}$ dessa amostra. No que diz respeito aos dados da primeira medida de $\mathrm{CO}, 38,5 \%(\mathrm{n}=42)$ tiveram índices graves de intoxicação, 37,6\% ( $\mathrm{n}=41)$ índices moderados e $16,5 \%(\mathrm{n}=18)$ índices leves. Os casos de missing somaram $7,3 \%(\mathrm{n}=8)$ dos resultados na primeira medida, com uma média de $18,48 \mathrm{ppm}(\mathrm{SD}=8,45)$ na primeira medida, que está situada na categoria de intoxicação moderada de acordo com os pontos de corte do instrumento. A segunda medida indicou índices médios de intoxicação por $\mathrm{CO}$ de $8,41 \mathrm{ppm}(\mathrm{SD}=6,14)$. Assim, em $82,2 \%(n=83)$ da amostra, o grau de intoxicação por $\mathrm{CO}$ foi moderado ou grave em primeira medida e apenas $22,7 \%(n=5)$ tiveram índices moderados ou graves na segunda medida.

Ainda em relação aos dados da segunda medida de $\mathrm{CO}$, levando-se em consideração não só os percentuais válidos, observou-se que apenas $1,8 \%(n=2)$ tiveram índices graves de intoxicação, 2,8\% (n=3) índices moderados e 15,6\% $(\mathrm{n}=17)$ índices leves. Os casos de missing somaram 79,8\% $(\mathrm{n}=87)$ dos resultados, posto que estão computados apenas os dados dos pacientes que aderiram ao tratamento. Levando-se em conta apenas os resultados do grupo considerado aderente, 9,1\% ( $\mathrm{n}=2)$ tiveram índices graves, 13,6\% ( $\mathrm{n}=3)$ índices moderados e 77,3\% ( $n=17)$ índices leves de intoxicação. 
Os índices de ansiedade e de depressão também foram avaliados. Os escores do BAI indicaram que 26,3\% ( $n=26)$ dessa amostra possuíam índices de ansiedade graves, $15,2 \%(\mathrm{n}=15)$ índices moderados, $32,3 \%(n=32)$ índices leves e 26,3\% ( $n=26)$ índices mínimos. O BAI teve uma média de 20,63 pontos $(\mathrm{SD}=14,65)$ que está situada na categoria leve, de acordo com os pontos de corte da escala. Já os escores do BDI, indicaram que $9,1 \%(\mathrm{n}=9)$ possuem índices graves de depressão, 29,3\% ( $\mathrm{n}=29)$ índices moderados, 22,2\% (n=22) índices leves e 39,4\% (n=39) índices mínimos, com uma média de 17,17 pontos $(\mathrm{SD}=11,21)$, que está situada na categoria leve de acordo com os pontos de corte da escala.

Os dados levantados pela aplicação da escala URICA demonstram que na primeira medida realizada $(\mathrm{n}=106)$, a sub-escala da "Pré-Contemplação" teve média de 13,11 ( $\mathrm{SD}=2,89)$, a "Contemplação" média de 29,41 ( $\mathrm{SD}=3,49)$, a "Ação" média de 28,89 ( $\mathrm{SD}=3,24)$ e a "Manutenção" média de 11,08 ( $\mathrm{SD}=6,56)$.

Comparando-se a aderência ao tratamento entre o grupo dos homens e das mulheres, pode-se observar que $40,0 \%(n=12)$ da amostra masculina foram aderentes à intervenção, enquanto que apenas $17,7 \%$ $(n=14)$ da amostra feminina foram consideradas aderentes, denotando que para efeitos desse estudo, os homens aderiram mais ao tratamento do que as mulheres $(p=0,023)$. Em relação às diferenças de níveis de ansiedade e de depressão, quando comparados homens e mulheres, pode-se observar que deve existir associação entre sexo e os escores do BAI $(p=0,003)$ e $\operatorname{BDI}(p=0,002)$, onde mais mulheres devem apresentar graus elevados de ansiedade e de depressão do que homens no início do tratamento.
Os escores iniciais de ansiedade dos pacientes que aderiram ao tratamento foram menores do que quando comparados àqueles que não aderiram $(p=0,023)$. As médias foram $15,50(\mathrm{SD}=11,76)$ e 22,45 pontos $(\mathrm{SD}=15,21)$, respectivamente. Os escores iniciais de depressão, igualmente, apresentaram-se menores entre àqueles que aderiram ao tratamento $(p=0,022)$. As médias foram de 12,42 pontos $(\mathrm{SD}=6,75)$ para os aderentes e 18,86 pontos $(\mathrm{SD}=12,01)$ para os que não aderiram, sugerindo que índices altos de ansiedade e de depressão são fatores que dificultam a aderência dos pacientes ao tratamento. Ademais, existe uma tendência corroborativa a esses dados, haja vista que os pacientes com escores mínimos de ansiedade aderiram mais e os mais graves aderiram menos à intervenção $(p=0,087)$. Já no tocante aos índices de depressão, pode-se dizer que também existe diferença estatisticamente significativa, posto que os mais deprimidos aderiram menos e os menos deprimidos demonstraram aderir mais $(p=0,007)$.

Com base nos resultados da amostra e nos pontos de corte do Monoxímetro, foram estabelecidas duas subcategorias entre os pacientes que aderiram ao tratamento $(\mathrm{n}=22)$, desprezando-se os casos de missing $(n=4)$. Aqueles que tiveram índices mínimos de intoxicação por $\mathrm{CO}$ foram considerados abstinentes, o que representou $77,3 \%(n=17)$ da amostra, e os que tiveram índices moderados ou graves de intoxicação por $\mathrm{CO}$, isto é, $22,7 \%(\mathrm{n}=5)$ dos pacientes, foram considerados ainda fumantes.

Os resultados obtidos através da aplicação da Escala URICA acerca dos Estágios Motivacionais estão apresentados na tabela abaixo.

Tabela 2: Transição dos Estágios Motivacionais nos quatro momentos

\begin{tabular}{lcccccc}
\hline $\begin{array}{l}\text { Medidas Estágio } \\
\text { Motivacional }\end{array}$ & N & $\begin{array}{c}\text { Média } \\
\text { Pontos }\end{array}$ & Desvio Padrão & $\begin{array}{c}\text { Pontuação } \\
\text { Mínima }\end{array}$ & $\begin{array}{c}\text { Pontuação } \\
\text { Máxima }\end{array}$ & P \\
\hline Pré-Contemplação 1 & 26 & 12,230 & 3,265 & 7 & 18 & \\
Pré-Contemplação 2 & 26 & 12,384 & 3,335 & 7 & 18 & 0,750 \\
Pré-Contemplação 3 & 26 & 12,269 & 3,219 & 7 & 18 & \\
Pré-Contemplação 4 & 26 & 12,269 & 3,329 & 7 & 20 & \\
Contemplação 1 & 26 & 30,115 & 2,916 & 27 & 35 & \\
Contemplação 2 & 26 & 30,000 & 2,800 & 26 & 35 & 0,903 \\
Contemplação 3 & 26 & 29,807 & 3,310 & 26 & 35 & \\
Contemplação 4 & 26 & 30,230 & 3,050 & 27 & 35 & \\
Ação 1 & 26 & 29,269 & 3,424 & 22 & 35 & \\
Ação 2 & 26 & 29,576 & 3,838 & 18 & 35 & 0,171 \\
Ação 3 & 26 & 29,846 & 3,196 & 24 & 35 & \\
Ação 4 & 26 & 30,269 & 2,793 & 28 & 35 & \\
Manutenção 1 & 26 & 9,961 & 5,264 & 7 & 24 & \\
Manutenção 2 & 26 & 18,461 & 8,324 & 7 & 31 & 0,000 \\
Manutenção 3 & 26 & 24,730 & 5,407 & 7 & 32 & \\
Manutenção 4 & 26 & 26,307 & 4,268 & 19 & 35 & \\
\hline Teste Friedman para comparação de médias com medidas repetidas. & & & & \\
& \multicolumn{7}{c}{}
\end{tabular}


Os resultados nos quatro momentos de aplicação demonstram que apenas a sub-escala do estágio de "Manutenção" teve modificação estatisticamente significativa $(\mathrm{p}<0,001)$. Além disso, os resultados indicam que essa diferença não está relacionada com os escores da FTND, que avalia o grau dependência de nicotina $(p=0,244)$. Outro dado importante relacionado aos resultados dessa escala é o de que os níveis de dependência de nicotina não estão relacionados à aderência ao tratamento $(p=0,687)$ e nem a categoria grave apresentou diferença estatisticamente significativa no tangente à aderência $(p=0,392)$.

\section{DISCUSSÃO}

A nicotina é uma droga de forte potencial de dependência. Apesar de demorar mais tempo do que outros tipos de estimulantes do sistema nervoso central para tornar o usuário dependente, tal como a cocaína, por exemplo, torna-se bastante difícil de interromper-se o uso após a dependência instalada (Longenecker, 1998). Os dados referentes ao grau de dependência de nicotina e níveis de intoxicação por $\mathrm{CO}$ dessa amostra demonstram o perfil de consumo e a gravidade do tabagismo nessa amostra. Contudo, não podem expressar claramente o grau de sofrimento que essas pessoas enfrentam diariamente na luta contra a terrível dependência do tabaco.

A média de tentativas prévias para se parar de fumar nessa amostra foi de 3,04 (SD=3,12). Essa dificuldade em ter sucesso na busca da abstinência está de acordo com o que está descrito na literatura, onde são necessárias em média de cinco a sete tentativas prévias para que se consiga atingir o objetivo (Achutti, 2001; Gigliotti e cols., 1999). Esses dados sugerem que, possivelmente, os pacientes que compõem esse estudo irão recair e realizar novas tentativas até conseguirem manter uma abstinência efetiva.

A porção feminina da amostra totalizava cerca de três quartos dos pacientes. Esses dados fazem pensar que, para fins desse estudo, as mulheres buscam mais tratamento, uma vez que dados epidemiológicos atuais dizem que $10,1 \%$ dos homens e $7,9 \%$ das mulheres são dependentes de nicotina no Brasil (Carlini, Galduróz, Noto \& Nappo, 2001). Assim, não se justifica essa predominância feminina na amostra, visto que os percentuais de dependência são semelhantes nos dois sexos. Entretanto, quando é comparada a aderência ao tratamento, pode-se observar que 40,0\% $(n=12)$ da amostra masculina foram aderentes à intervenção, enquanto que apenas $17,7 \%(\mathrm{n}=14)$ da amostra feminina foram consideradas aderentes, denotando que para efeitos desse estudo, os homens aderiram mais ao tratamento do que as mulheres $(p=0,023)$. Isso talvez se deva ao fato de que a porção masculina da amostra demonstrou ser menos ansiosa $(p=0,003)$ e menos deprimida $(p=0,002)$ do que quando comparada às mulheres que compuseram o estudo.

O BAI e o BDI tiveram suas médias de pontos situadas na categoria leve, de acordo com os pontos de corte das escalas. Os escores de ansiedade dos pacientes que aderiram ao tratamento foram menores do que quando comparados àqueles que não aderiram $(p=0,023)$. Os escores de depressão igualmente apresentaram-se menores entre àqueles que aderiram ao tratamento $(p=0,022)$. Esses dados sugerem que índices altos de ansiedade e de depressão são fatores que dificultam a aderência dos pacientes ao tratamento, o que corrobora com achados importantes de outros estudos (Bull, Burke, Walsh \& Whitehead, 2003; Hitsman e cols., 2002).

Com base nos dados obtidos pelo uso do Monoxímetro, pode-se fazer uma avaliação objetiva da abstinência dos pacientes que concluíram o tratamento. Aqueles que tiveram índices mínimos de intoxicação por $\mathrm{CO}$ foram considerados abstinentes, o que representou 77,3\% ( $n=17)$ da amostra, e os que tiveram índices moderados ou graves de intoxicação por $\mathrm{CO}$, isto é, $22,7 \%(n=5)$ dos pacientes, foram considerados ainda fumantes.

Os resultados obtidos pela aplicação da Escala URICA nos quatro momentos demonstram que apenas a sub-escala do estágio de "Manutenção" teve modificação estatisticamente significativa $(\mathrm{p}<0,001)$, ou seja, os pacientes mantiveram as mesmas pontuações nas sub-escalas da "Pré-Contemplação" $(p=0,750)$, "Contemplação" $(p=0,903)$ e "Ação" $(p=0,171)$ do início ao fim da intervenção.

Não obstante a isso, há que se ter cautela na análise desses dados, uma vez que a maioria dos pacientes, o correspondente a 76,1\% ( $\mathrm{n}=83)$, não foram submetidos a todas as quatro medidas da Escala URICA, em face de que não completaram a intervenção. Dessa forma, os dados possivelmente não teriam sido exatamente os mesmos se todos os 109 pacientes tivessem sido submetidos à avaliação completa, isto é, aos quatro momentos, e não somente os $23,9 \%(\mathrm{n}=26)$ considerados aderentes, como de fato ocorreu.

Os resultados da Análise Multivariada indicam que a diferença na sub-escala da Manutenção não está 
relacionada com os escores da FTND, que avalia o grau dependência de nicotina $(p=0,244)$. Nessa sorte, para fins desse estudo, o grau de dependência de nicotina não interfere na modificação do estágio motivacional, no que se refere ao tratamento do tabagismo. Outro dado importante relacionado aos resultados dessa escala é o de que os níveis de dependência de nicotina também não estão relacionados à aderência ao tratamento $(p=0,687)$ e nem a categoria grave apresentou diferença estatisticamente significativa no que diz respeito à aderência ou não-aderência $(p=0,392)$. Nesse sentido, para fins desse estudo, ser mais ou menos dependente não é fator de sucesso ou fracasso na aderência à intervenção.

\section{CONCLUSÃO}

Dessa forma, pode-se dizer que o tabagismo é uma grave mazela social existente em todo o mundo e uma das dependências químicas de mais difícil tratamento (Achutti, 2001). Um dos principais fatores para essa dificuldade é, sem sombra de dúvidas, a motivação, entendida como um estado de avidez e de prontidão para a mudança (Miller \& Rollnick, 2001). Entretanto, algumas outras variáveis também demonstraram serem importantes no que diz respeito aos índices de aderência ao tratamento tais como os níveis dos sintomas de ansiedade e de depressão, o que está de acordo com algumas importantes publicações acerca do tratamento do tabagismo (O'Connell e cols., 2003; Fergusson e cols., 2003). De acordo com os resultados do presente estudo, quanto maiores os escores de ansiedade e de depressão, menor será a aderência ao tratamento.

Ainda com relação à aderência ao tratamento, pode-se observar que os homens que participaram desse estudo aderiram mais do que quando comparado às mulheres, o que está de acordo com outros estudos ligados a aderência ao tratamento do tabagismo (Borrelli, Papandonatos, Spring, Hitsman \& Niaura, 2004). Outro dado surpreendente relacionado aos resultados foi o fato de que a pontuação da FTND, que avalia o grau de dependência de nicotina, não apresentou relação quanto à aderência ao tratamento.

No que incide aos Estágios Motivacionais dos pacientes que iniciaram e concluíram a intervenção, isto é, àqueles considerados aderentes, vislumbra-se que eles iniciam e concluem o tratamento com os mesmos escores, no que diz respeito aos estágios de "PréContemplação", "Contemplação" e "Ação". Entretanto, quando se trata do estágio da "Manutenção", observou-se que os escores dessa sub-escala da URICA vão aumentando, significativamente, ao longo da intervenção.

Os índices de abstinência da parcela da amostra que concluiu o tratamento, ou seja, que foi considerado aderente, mostrou-se alto. Contudo, o acompanhamento foi de apenas oito semanas, o que representa pouco tempo para se ter certeza de que a abstinência será efetiva. Sugere-se um estudo de follow-up para que se possa observar se a abstinência de praticamente três quartos da amostra irá manter-se durante os próximos meses.

Corolário de tudo isso, quanto mais se aprender sobre os estágios motivacionais, e a própria dependência de tabaco, melhor capacitados estaremos para o tratamento dos nossos pacientes. Isso, sem qualquer óbice, poderá acrescentar muito na qualidade de nossa prática profissional, bem como no alcance de resultados mais positivos do que aqueles encontrados pelos dos tratamentos hoje empregados no tratamento do tabagismo.

\section{REFERÊNCIAS}

Achutti, A. (2001). Guia nacional de prevenção e tratamento do tabagismo. Rio de Janeiro: Vitrô Comunicação \& Editora.

Beck, A. T. \& Steer, R. A. (1993a). Beck Anxiety Inventory Manual. San Antonio: Psychological Corporation.

Beck, A. T. \& Steer, R. A. (1993b). Beck Depression Inventory Manual. San Antonio: Psychological Corporation.

Bedfont Scientific. (1993). Operator's manual for mini and micro smokerlyzers. Upchurch: Bedfont Scientific.

Borrelli, B., Papandonatos, G., Spring, B., Hitsman, B. \& Niaura, R. (2004). Experimenter-defined quit dates for smoking cessation: Adherence improves outcomes for women but not for men. Addiction, 99(3), 378-385.

Bull, L., Burke, R., Walsh, S. \& Whitehead, E. (2003). Smoking in pregnancy and parenthood: What is the role of depression, anxiety and nicotine addiction? Early Child Development and Care, 173(2/3), 349-356.

Burke, B. L., Arkowitz, H. \& Menchola, M. (2003). The efficacy of motivational interviewing: A meta-analysis of controlled clinical trials. Journal of Consulting and Clinical Psychology, 71(5), 843-861.

Carey, K. B., Purnine, D. M., Maisto, S. A. \& Carey. M. P. (1999). Assessing readiness to change substance abuse: A critical review of instruments. Clinical Psychology: Science and Practice, 6, 245-266.

Carlini, E. A, Galduróz, J. C. F., Noto, A. R. \& Nappo, S. A. (2001). I Levantamento domiciliar sobre o uso de drogas psicotrópicas no Brasil: Estudo envolvendo as 107 maiores cidades do país. São Paulo: Cebrid - Centro Brasileiro de Informações Sobre Drogas Psicotrópicas: Unifesp - Universidade Federal de São Paulo. 
Carmo, J. T. \& Pueyo, A. A. (2002). A adaptação ao português do Test for Nicotine Dependence (FTND) para avaliar a dependência e tolerância à nicotina em fumantes brasileiros. Revista Brasileira de Medicina, 59(1/2), 73-80.

Cunha, J.A. (2001). Manual da versão em português das Escalas Beck. São Paulo: Casa do Psicólogo.

Di Clemente, C. C. \& Prochaska, J. O. (1985). Processes and stages of change of change: Coping and competence in smoking behavior change. Em S. Shiffman \& T. A. Wills (Orgs.), Coping and substance abuse (pp. 319-342). New York: Academic Press.

Fagerström, K. O. (1978). Measuring degree of physical dependence to tobacco smoking with reference to individualization of treatment. Addictive Behaviors, 3, 235-241.

Fergusson, D. M, Goodwin, R. D. \& Horwood, L. J. (2003). Major depression and cigarette smoking: Results of a 21-year longitudinal study. Psychological Medicine, 33(8), 1357-1367.

Figlie, N. B. (1999). Motivação em alcoolistas tratados em Ambulatório Específico para Alcoolismo e em Ambulatório de Gastroenterologia. Dissertação de Mestrado. Universidade Federal de São Paulo, Escola Paulista de Medicina. São Paulo, SP.

Gigliotti, A., Bonetto, D. V. S., Alves, J. G., Jardim, J. R. B., Maranhão, M. F. C. \& Zamboni, M. (1999). Tabagismo. Jornal Brasileiro de Medicina, 77(2), 01-16.

Gigliotti, A., Carneiro, E. \& Ferreira, M. (2001). Tratamento do Tabagismo. Em B. Rangé (Org.), Psicoterapias cognitivocomportamentais: Um diálogo com a psiquiatria. Porto Alegre: Artmed.

Heather, N. (1992). Addictive disorders are essentially motivational problems. British Journal of Addiction, 87, 828-830.

Hitsman, B., Abrams, D. B., Shadel, W. G., Niaura, R., Borrelli, B. \& Emmons, K. M. (2002). Depressive symptoms and readiness to quit smoking among cigarette smokers in outpatient alcohol treatment. Psychology of Addictive Behaviors, 16(3), 264-268.

Longenecker, G. L. (1998). Como agem as drogas. São Paulo: Quark Books.

Mc Connaughy, E. A., Di Clemente, C. C., Prochaska, J. O. \& Velicer, W. F. (1983). Stages of change in psychotherapy: A follow-up report. Psychotherapy, 26, 494-503.

Miller, W. \& Rollnick, S. (2001). A entrevista motivacional: Preparando as pessoas para mudança de comportamentos aditivos. Porto Alegre: Artes Médicas.

O’Connell, M., Lucan, S. C., Yeh, M. C., Rodríguez, E., Shah, D. \& Chan, W. (2003). Impediment profiling for smoking cessation: Results of a pilot study. American Journal of Health Promotion, 17(5), 300-303.

Pollak, K. I., Carbonari, J. P, Di Clemente, C. C., Niemann, Y. F. \& Mullen, P. D. (1998). Causal relationships of process of change and decisional balance: Stage-specific models for smoking. Addictive Behaviors, 23(4), 437-448.

Prochaska, J. O. \& Di Clemente, C. C. (1982). Transtheoretical therapy: Toward a more integrative model of change. Psychotherapy: Theory, Research and Practice, 19, 276-288.

Sutton, S. (1997). Os Estágios de Mudança podem orientar o tratamento das adições? Um exame crítico do Modelo de Prochaska e Di Clemente. Em G. Edwards \& C. Dare (Orgs.), Psicoterapia e tratamento de adições (pp. 173-188). Porto Alegre: Artes Médicas.

\section{Sobre os autores:}

Wilson Vieira Melo: Psicólogo, Mestre em Psicologia Clínica (PUCRS), Professor e Supervisor do Curso de Especialização em Psicoterapia Cognitivo-Comportamental da WP - Centro de Psicoterapia Cognitivo Comportamental, Professor de Graduação em Psicologia na FACCAT - Faculdades de Taquara.

Margareth da Silva Oliveira: Psicóloga, Doutora em Ciências (UNIFESP), Professora do Programa de Pós-graduação e de Graduação em Psicologia da PUCRS - Pontifícia Universidade Católica do Rio Grande do Sul.

Elisa Arrenti Ferreira: Psicóloga, aluna do Curso de Especialização em Psicoterapia Cognitivo-Comportamental da WP - Centro de Psicoterapia Cognitivo-Comportamental.

Endereço para correspondência: Wilson Vieira Melo - Rua Santa Cecília, 1.556 - Bairro Santa Cecília - 90.420-040 - Porto Alegre/RS - Endereço eletrônico: melo@ terapiascognitivas.com.br. 